\title{
La competencia instrumental específica en el grado de Administracion y Dirección de Empresas de la Universitat Politècnica de València
}

\author{
Ribal, Javier ${ }^{\mathrm{a}}$; Andrés, Silvia ${ }^{\mathrm{b}}$; Blasco, Ana ${ }^{\mathrm{c}}$; Cervelló, Roberto ${ }^{\mathrm{d}}$; Chirivella, \\ Vicente $^{\mathrm{e}}$ \\ ${ }^{a}$ Dep. Economía y Ciencias Sociales. UPV. frarisan@upv.es. ${ }^{b}$ Dep. Economía y Ciencias Sociales. UPV. \\ silangle@esp.upv.es. ${ }^{\mathrm{c} D e p}$. Economía y Ciencias Sociales. UPV. ablascor@upvnet.upv.es. ${ }^{\mathrm{d} D e p}$. Economía y Ciencias \\ Sociales. UPV. rocerro@esp.upv.es. ${ }^{e}$ Departamento de Estadística e Investigación Operativa Aplicadas y Calidad. \\ UPV.vchirive@eio.upv.es
}

\begin{abstract}
The soft skill termed as specific instrumental skill deals with using tools and technologies needed in the work environment. The word "specific" is referred to the specific degree. Its goal should be closing the gap between the skills and capabilities of graduates, and the requirements and demands of the work environment. In the Business Administration School at the Universidad Politècnica de València several courses assess this soft skill considering the spreadsheet software as the tool needed in the work environment while others build models by means of open-source software.
\end{abstract}

Keywords: soft skills, work requeriments, spreeadsheet software, business administration degree

\section{Resumen}

La competencia transversal instrumental especifica hace referencia al uso de herramientas y tecnologías necesarias para el ejercicio profesional. El término especifico hace referencia a la titulación. El objetivo de la misma debe ser aproximar las competencias y habilidades de los graduados a los requerimientos y demandas de las empresas. En la Facultad de Administración y Dirección de Empresas de la Universitat Politècnica de València varias de las asignaturas que evalúan esta competencia lo hacen empleando la hoja de cálculo como la herramienta necesaria para el ejercicio profesional. Otras se centran en técnicas y modelos específicos de la materia mediante el uso de software libre.

Palabras clave: competencias transversales, demandas laborales, hoja de cálculo, grado en Direccion y Administración de Empresas 


\section{Introducción}

La competencia transversal "instrumental específica" hace referencia al uso de las herramientas y tecnologías necesarias para el ejercicio profesional asociado a cada titulación. De acuerdo con el proyecto de competencias transversales de la Universitat Politècnica de València (UPV) el estudiante será capaz de identificar las herramientas más adecuadas en cada caso, conociendo sus utilidades y siendo capaz de integrarlas y combinarlas para poder resolver un problema, realizar un proyecto o un experimento. Esta definición adaptada a los estudios de graduado en Administración y Dirección de Empresas se puede expresar como identificación de modelos, técnicas y software más adecuados en cada caso. Tickle et al (2014) concluyen que los graduados no muestran tanta competencia en el uso de software como los empleadores quisieran. Los autores atribuyen la falta de competencia a la carencia en formación coherente en las universidades. Andrews y Higson (2008) también citan la existencia de preocupación sobre las amplias diferencias entre las competencias y capacidades de los graduados y los requerimientos y demandas laborales. Por ello la competencia transversal instrumental específica resulta importante para la inserción labora de los graduados en general y de los graduados de ADE en particular. Jones et al (2017) señalan que las competencias transversales pueden suponer una ventaja competitiva para las universidades físicas frente a las universidades on-line.

El objetivo de este trabajo es conocer el funcionamiento y evaluación de la competencia instrumental específica en el grado de Administración y Dirección de Empresas de la UPV así como proponer mejoras y detectar posibilidades de coordinación entre las asignaturas.

\section{La competencia instrumental especifica en el grado de ADE}

En los grados de la UPV las competencias transversales se evaluán en dos niveles de dominio, 1 y 2 . El nivel 1 se valora en asignatura de los dos primeros años, mientras que el nivel 2 se evalora en asignaturas de los años 3 y 4 del grado.

Para la competencia transversal instrumental especifica, el nivel 1 se concreta en "Emplear correctamente las herramientas básicas del ámbito profesional de forma guiada" y tiene como indicadores:

- Identificar las herramientas básicas y su utilidad.

- Manejar las herramientas básicas siguiendo unas instrucciones previamente dadas.

- Hacer uso seguro y responsable de las herramientas.

El nivel 2 se concreta en "Integrar correctamente las herramientas básicas del ámbito profesional de forma autónoma" y tiene como indicadores:

- Manejar las herramientas básicas de forma autónoma. 
- Seleccionar y combinar las herramientas básicas adecuadas para realizar un proyecto y/o resolver un problema complejo.

Existe un nivel 3 si bien éste no se valora en los estudios de grado.

Para el desarrollo de esta competencia el proyecto institucional de la UPV sobre competencias transverales recomienda actividades formativas como prácticas de laboratorio, problemas, proyectos y portafolio. Como procedimiento de evaluación las rúbricas son la principal herramienta sugerida.

Los puntos de control (asignaturas) se reflejan en la tabla 1 junto con el nivel dominio. Todas ellas son asignaturas obligatorias por lo que todos los alumnos del grado las cursan. La existencia de los puntos de control no implica que la competencia no pueda ser obtenida también en otras asignaturas, como es probable que suceda.

Tabla 1. Puntos de control competencia transversal instrumental específica

\begin{tabular}{lcc}
\hline \multicolumn{1}{c}{ Asignatura } & Curso & Nivel de dominio \\
\hline Introducción a las finanzas & $1^{\mathrm{o}}$ & 1 \\
Matemáticas financieras & $1^{\mathrm{o}}$ & 1 \\
Microeconomía & $1^{\mathrm{o}}$ & 1 \\
Econometría & $2^{\mathrm{o}}$ & 1 \\
Economía financiera & $3^{\mathrm{o}}$ & 2 \\
\hline
\end{tabular}

A continuación se desarrolla el tratamiento de la competencia en cada uno de los puntos de control.

\section{La competencia instrumental específica en Introducción a las finanzas}

La asignatura de Introducción a las finanzas es una asignatura básica situada en el primer año en el primer cuatrimestre y por tanto los estudiantes acaban de acceder a la universidad cuando cursan la asignatura. Se trata de una asignatura introductoria que combina las dos partes fundamentales de las finanzas corporativas: inversión $\mathrm{y}$ financiación. En ambas partes la herramienta estándar es la hoja de cálculo, habitualmente Microsoft Excel. El uso de la hoja de cálculo es habitual en todos los entornos corporativos independientemente del tamaño y del sector por ello es un elemento conductor de la asignatura, no sólo de la asignatura sino del resto de asignaturas de naturaleza financiera del grado de ADE.

Al tratarse de alumnos de primer año su conocimiento previo del funcionamiento básico de una hoja de cálculo es muy desigual. Algunos estudiantes no han tenido ningún contacto previo con la hoja de cálculo mientras que otros están totalmente familiarizados con la realización de cálculos sistemáticos y la fijación de celdas. Los objetivos a conseguir en cuento a la competencia por parte de los alumnos son varios: toma de 
contacto y comprensión del potencial de la hoja de cálculo, consciencia del uso continuado que harán de la misma tanto en el grado como profesionalmente, adopción de buenas prácticas en la modelización financiera, iniciación con gráficos y funciones financieras. Asimismo, con la competencia también se busca igualar el nivel de manejo de la hoja de cálculo entre los estudiantes, que seguirán empleándola de forma recurrente a lo largo del grado.

Los alumnos realizan siete prácticas de dos horas en laboratorio informático a lo largo del cuatrimestre. Las prácticas son aplicaciones en hoja de cálculo de problemas y conceptos previamente estudiados a nivel teórico y en ejercicios en aula. De modo progresivo se van introduciendo funcionalidades de la hoja de cálculo de modo que el alumno comprende la capacidad de realizar cálculos sistemáticos de un modo inmediato así como la posibilidad de variar los datos de entrada evaluando el cambio en los resultados.

Las seis primeras prácticas son evaluadas individualmente y el estudiante sube su trabajo a una tarea específica creada en PoliformaT. El trabajo es individual o en parejas, dependiendo del número de estudiantes del grupo, se fomenta el trabajo individual pero por problemas de estructura no siempre es posible. El trabajo realizado se evalúa mediante un test en poliformaT para comprobar que el alumno ha realizado correctamente la práctica.

La séptima práctica es un examen individual de una hora en la que el alumno tiene que aplicar lo aprendido y prácticado en las prácticas previas. La nota del examen y de las prácticas anteriores contribuyen a la nota final de la asignatura y simultáneamente la nota del examen se convierte en un nivel de consecución de la competencia. Lo solicitado en cuanto a nivel de consecución se adecua al nivel 1 fijado para la competencia: "Emplear correctamente las herramientas básicas del ámbito profesional de forma guiada".

\section{La competencia instrumental específica en Matemáticas financieras}

En el caso de matemáticas financieras, se programan unas sesiones de prácticas que abordan la resolución de verdaderos problemas de finanzas con la hoja de cálculo y al nivel de dificultad establecido en la asignatura de acuerdo a sus contenidos.

Se tratar de utilizar las prácticas para el planteamiento de verdaderos problemas y no la mera resolución de ejercicios prácticos, en grupos numerosos que han de compartir los recursos informáticos del aula, de forma que al resolver los problemas de docencia, se incorpore la competencia CT-13 "Instrumental específico" que favorezca un mejor proceso de aprendizaje.

La competencia, CT-13 "Instrumental específica" se implementan a través de las 7 sesiones de prácticas previstas.

Con el objeto de incorporar dicha competencia: 
- Se presta una especial atención al procedimiento de resolución, introduciendo en el conocimiento de herramientas para dicha resolución, así como la interpretación y el análisis de los resultados de las mismas.

- Se utilizan las tecnologías más avanzadas en relación al ámbito profesional para el que se prepara el alumno y de acuerdo con los conocimientos y las competencias profesionales que debe adquirir

Por otra parte y de acuerdo con la CT-13 la resolución de casos y supuestos se realizan con instrumental específico, en concreto la hoja de cálculo y otras herramientas ofimáticas, que el alumno tendrá a su disposición en la sesión. En algunos casos, se proponen plantillas para la resolución de los problemas, a fin de ayudar al alumno a identificar los datos, las cuestiones que se plantean y la sistemática para la obtención de resultados que permitan el análisis, las conclusiones sobre los resultados y en definitiva la toma de decisiones a partir de los mismos.

En las sesiones de prácticas los grupos realizan un trabajo tutelado por el profesor, desarrollando cada grupo sus propias herramientas informáticas para la resolución del ejercicio, en Excel. La Hoja de cálculo se archiva en el espacio compartido, desde donde estará disponible para cada alumno y para los profesores de la asignatura.

En las sucesivas sesiones, el grupo irá creando su propio libro de excel, que se conformará a modo de plantilla de análisis, válida para el análisis de distintas situaciones y problemas, con el objeto de que el alumno cree una herramienta de análisis útil para su desarrollo profesional futuro.

Como elementos de guía para el profesor, en cada práctica se sigue la siguiente estructura:

- Explicación dinámica de trabajo en cada sesión

- Lectura inicial enunciado. Repasar los conceptos vistos en las unidades. Identificación de datos y cuestiones a resolver.

- Indicar al alumno las pautas para la elaboración-conformación de la hoja de cálculo:

- formato,

- identificación de datos,

- definición de área para los resultados,

- funciones y operaciones de cálculo a realizar

- nombrar hojas adecuadamente, ...

- Resolver dudas durante el desarrollo de la práctica

La evaluación se realiza comprobando el correcto diseño de la hoja de cálculo y se otorga una calificación numérica. Hasta el momento no se han empleado rúbricas puesto que los alumnos disponen de una plantilla de orientación al comienzo de la práctica. 


\section{La competencia instrumental específica en Microeconomía I}

En la asignatura de Microeconomía I la competencia instrumental específica se estructura entorno a dos clases prácticas de laboratorio informático, garantizando que los estudiantes sepan acceder y manejarse con bases de datos de carácter económico, uso de procesador de texto y hoja de cálculo para preparar su informe, con realización de los cálculos correspondientes de carácter microeconómico, elaboración de tablas y gráficas, argumentación/justificación microeconómica, conclusiones, bibliografía. Así, el estudiante debe identificar las herramientas más adecuadas en cada caso, conociendo sus utilidades, su funcionamiento y siendo capaz de integrarlas y combinarlas para poder resolver el caso planteado.

La evaluación se realiza a partir de los informes de prácticas que los alumnos deben elaborar con carácter individual. Los indicadores de evaluación utilizados son los siguientes:

- Definir el problema describiendo de manera clara y concisa los aspectos más importantes. Estructura del texto.

- Utilizar con soltura fuentes de información y bases de datos telemáticas y seleccionar los datos correctos.

- Emplear correctamente herramientas analíticas e informáticas para la resolución del problema o caso propuesto.

- Analizar y argumentar la coherencia de las soluciones obtenidas.

\section{La competencia instrumental específica en Econometría}

Econometría es una asignatura básica, que está ubicada en segundo curso del grado de ADE, en tercer curso en la doble titulación de ADE con Informática, y en cuarto curso en la doble titulación de ADE con Telecomunicaciones. El punto de control sobre la competencia transversal 13, instrumental específica, se realiza a su nivel I, pese a que la asignatura se imparte en cursos en los que se debería evaluar a nivel II.

En el contexto de la asignatura, la competencia que deben adquirir los alumnos consiste en la resolución de problemas reales mediante el modelo más adecuado para ello y el uso de software de métodos cuantitativos, que es específico para la materia, y de libre uso, por lo que los alumnos pueden seguir utilizándolo en su trabajo sin que sea necesaria la adquisición de licencia. La resolución de los problemas abarca desde la obtención de datos en bases de datos públicas y en memorias anuales de grandes empresas, la identificación del modelo que permitirá su análisis, el manejo de un software específico para su estimación, y la interpretación del análisis realizado, con especial cuidado de realizar dicha interpretación de forma que pueda ser entendida "por cualquier miembro de la empresa". Todo ello debe quedar plasmado en una memoria, que se utilizará en la valoración de la competencia. 
Más específicamente, la evaluación se hará por medio de preguntas de respuesta abierta, promediando los resultados de las doce evaluaciones realizadas a lo largo del curso, y reconvirtiendo el valor numérico en los niveles establecidos para valorar la competencia. Parece razonable el uso de la nota promedio como indicador del nivel adquirido, porque la identificación de la herramienta, la realización del análisis y la interpretación de los resultados es precisamente el objetivo la sesión de prácticas.

Finalmente, la competencia de los alumnos en cuanto al manejo del software se realiza mediante la observación directa por parte del profesor, sin una prueba específica. Como todos los alumnos terminan por adquirir la habilidad del manejo del programa de ordenador, tras doce semanas de uso, ésta no se evalúa explícitamente.

\section{La competencia instrumental específica en Economía Financiera}

Al igual que en las asignaturas previas de naturaleza financiera, Introducción a las Finanzas y Matemáticas Financieras la competencia transversal instrumental específica vuelve a tener como eje la hoja de cálculo. En este caso el nivel de dominio es el 2, por lo que se espera del estudiante un mayor dominio de la herramienta que le permita incorporarse al mercado laboral.

La asignatura de Economía Financiera profundiza en el análisis de inversiones, tanto financieras como reales y en la misma se aplican diversos métodos y técnicas a fin de tomar decisiones en la selección de inversiones. Específicamente, el objetivo de la asignatura es que el estudiante conozca y comprenda los principios fundamentales de la ciencia de la inversión y que los aplique para realizar estimaciones y cálculos prácticos que conduzcan a decisiones de inversión correctas. Es decir, la componente cuantitativa es una herramienta para facilitar la toma de la decisión final “invertir o no invertir". Esa componente cuantitativa se realiza mediante la hoja de cálculo en cinco prácticas de laboratorio de 2 horas cada una. En ellas el alumno debe realizar un ejercicio relacionado con el análisis de inversiones financieras o reales. Se parte del conocimiento previamente adquirido por los alumnos y se profundiza en funciones financieras y técnicas de optimización y simulación para la toma de decisiones financieras. Se incide en la distinción de datos de entrada y resultados y en la capacidad de la hoja de cálculo de rehacer todos los cálculos si el diseño es el adecuado. Asimismo, se continua con la creación de gráficos y figuras ya comenzada en la asignatura de Introducción a las finanzas.

Los alumnos deben subir el trabajo realizado, preferentemente de forma individual, a PoliformaT. Durante la práctica el profesor hace seguimiento del trabajo de los alumnos. El peso de las prácticas es el mismo para todas ellas y la nota final es la media de la nota númerica. Esta nota numérica es parte de la calificación de la asignatura y además es convertida en un nivel de consecución de la competencia (A, B, C o D). 


\section{Discusión y conclusiones}

La competencia transversal $n^{0} 13$, Instrumental Específica, es una competencia transversal diferente del resto por definición, ya que mezcla el concepto transversal con el concepto específico. Sin embargo, si se define adecuadamente, alrededor de asignaturas o software de naturaleza transversal, que se empleen en el mercado laboral sin diferenciar sector o tamaño de empresa puede resultar muy útil para el curriculum y futuro profesional del estudiante. La literatura señala la existencia de diferencias en las materias enseñadas en las universidades y las demandas de las empresas por lo que las competencias transversales en general y la competencia instrumental específica deberían ayudar a reducir esas diferencias.

Tres de los puntos de control utilizan la hoja de cálculo como eje de la competencia transversal, lo cual es coherente considerando que este tipo de software se emplea de un modo universal en los entornos corporativos. Formby et al (2017) muestran la necesidad de los estudiantes de Administración de Empresas de poseer competencias en hoja de cálculo, específicamente en Microsoft Excel y también el requerimiento de las mismas por parte de las empresas. En este sentido es acertado el diseño de la competencia entorno a la hoja de cálculo. El inventario de uso de software en el grado de ADE indica que la hoja de cálculo es mayoritariamente el software más utilizado en prácticas de laboratorio. Concretamente se imparten 85 horas en prácticas de laboratorio con hoja de cálculo en asignaturas obligatorias y en torno a 10 más en cada una de las intensificaciones.

Pese a todo, hay necesidades de software más específicas que pueden ser cubiertas mediante el uso de programas libres. De un tiempo a esta parte el software libre va aumentando su uso a medida que amplía sus prestaciones y gana popularidad. Entre otras ventajas, el software libre elimina los costes de licencias, facilita que los alumnos trabajen en sus casas (de forma legal) con las mismas herramientas informáticas que utilizan en la facultad, crea comunidades que comparten conocimientos, permite el trabajo en cualquier sistema operativo y con ordenadores de potencia media, ... y el egresado puede trasladar estas ventajas a la propia empresa, más adelante.

Por otro lado, se detecta falta de coordinación, una estrategia a nivel del grado entre las asignaturas y la competencia. A nivel de facultad sería conveniente tener una estrategia para la competencia que siga líneas específicas como pueden ser distintos tipos de software, no sólo en las asignaturas punto de control sino en todas aquellas que empleen el mismo software.

En cuanto a la evaluación de las mismas si bien las rúbricas son la herramienta sugerida no es prácticamente utilizada por las asignaturas punto de control. 


\section{Referencias}

ANDREWS, J, HIGSON, H. (2008). "Graduate employability,'soft skills' versus 'hard'business knowledge: A European study." Higher education in Europe vol 33, no 48, p 411-422.

FORMBY, S. K., MEDLIN, D., ELLINGTON, V. B. (2017)“Microsoft Excel®: is it an important job skill for college graduates?". Information Systems Education Journal, vol. 15, no 3, p. 55.

JONES, M., BALDI, C., PHILLIPS, C., WAIKAR, A. (2017). "The hard truth about soft skills: What recruiters look for in business graduates". College Student Journal, vol. 50, no 3 , p 422-428.

TICKLE, L., KYNG, T., WOOD, L. N. (2014) "The role of universities in preparing graduates to use software in the financial services workplace". International Journal of Mathematical Education in Science and Technology, vol. 45, no 2, p. 200-213. 